\title{
Evaluación de la abundancia relativa y percepción de la presencia de palomas Columba livia (Columbiformes: Columbidae) en la Universidad Nacional de Costa Rica
}

\author{
Evaluation of Relative Abundance and Perception of the Presence of Rock Pigeons \\ Columba Livia (Columbiformes: Columbidae) at the National University of Costa Rica
}

Óscar Ramírez-Alán

osoramirez@gmail.com

Escuela de Ciencias Biológicas

Universidad Nacional

Heredia, Costa Rica

Jorge De la O-Castro

mdelao29@gmail.com

Escuela de Ciencias Biológicas

Universidad Nacional

Heredia, Costa Rica

Dayatri Bolaños-Picado

dayab19@hotmail.com

Escuela de Ciencias Biológicas

Universidad Nacional

Heredia, Costa Rica

Jean Karl Mc Queen-Blanco

jeankmcqueen@gmail.com

Escuela de Ciencias Biológicas

Universidad Nacional

Heredia, Costa Rica

Recibido-Received: 10/dic/2015 / Corregido-Corrected: 15/jun /2016.

Aceptado-Accepted: 20/ago/2016 / Publicado-Published: 31/ene /2017.

\begin{abstract}
Resumen
La presencia de la paloma Columba livia en las obras de infraestructura conlleva a un deterioro de estas mismas; el aumento de estas aves en grandes ciudades ha alarmado a la población, igual situación ocurre en las instalaciones de la Universidad Nacional. El objetivo de este trabajo consistió en monitorear la población para conocer la abundancia relativa. Para ello, se seleccionaron cinco edificios dentro de la Universidad, cuyo criterio de selección fue la presencia de palomas. Se realizaron observaciones directas durante un periodo de cinco meses, tres veces por semana. Se encontró que el costado oeste de Química es el más concurrido (4.37 \pm 1.17$)$, utilizan, ahí, espacios como los equipos
\end{abstract}


UNICIENCIA Vol. 31, No. 1, pp. 29-38. Enero-junio, 2017.

ISSN Electrónico: 2215-3470

URL: www.revistas.una.ac.cr/uniciencia

DOI: $\underline{\text { http://dx.doi.org/10.15359/ru.31-1.4 }}$

Email: revistauniciencia@una.cr

para el aire acondicionado y agujeros en los techos como sitios para anidación. Un 53\% de personal de la Universidad encuestado está en desacuerdo en procesos de erradicación. La disponibilidad de infraestructura y los recursos alimentarios son factores ecológicos claves para atraer palomas, ya que favorecen sitios para la anidación y descanso, lo que contribuye directamente en el crecimiento de sus poblaciones. Se recomienda reducir los sitios artificiales de percha, tapar entradas existentes que puedan utilizarse para la anidación y educar a la población acerca de los problemas potenciales que representa el incremento poblacional de las palomas.

Palabras claves: Incremento; percha; abundancia relativa; percepción social; Universidad Nacional.

\begin{abstract}
The presence of Columba livia dove in infrastructures leads to their deterioration. The increase of these birds in large cities has alarmed the population; the same situation occurs at the National University of Costa Rica premises. This study aimed to monitor the population due to its relative abundance. Five buildings within the university were selected, following the criterion of the presence of pigeons. Direct observation was conducted over a period of five months, three times a week. It was found that the west side of the Chemistry building is the most crowded ( $4.37 \pm 1.17)$; the birds use spaces, such as air conditioners and holes on the roofs, as sites for nesting. 53\% of the University workers interviewed disagreed in eradication processes. The availability of food resources and infrastructure are key factors to attract pigeons as these factors are ideal for nesting and roosting, which directly contributes to the growth of their populations. We recommend reducing artificial sites that can be used as perch, covering all the possible holes used for nesting, and educating people about potential problems that these pigeons can create.
\end{abstract}

Keywords: Increase; perch; relative abundance; social perception; National University.

La paloma Columba livia (paloma doméstica, conocida comúnmente como paloma de Castilla) es una especie residente del territorio costarricense. Corresponde a una especie introducida en Costa Rica proveniente de Europa.

Diversos estudios identificaron un aumento en la cantidad de individuos de C. livia al mismo tiempo que existía un auge en el desarrollo de obras de infraestructura en los centros de las ciudades, lo cual ocasionó que vieran en tales obras un hábitat propicio para su diario vivir (Jokimaki et al., 1998). Lo anterior es evidente en la gran área metropolitana, donde el uso que las palomas le han dado a los edificios, en muchas ocasiones con un gran valor cultural (e.g. Teatro Nacional) ha conllevado a un notable deterioro sobre las edificaciones (Mendez et al., 1995).

La paloma C. livia se ha convertido en una especie plaga para cierta población de costarricenses mientras que para otra parte posee un gran valor cultural y natural en el entorno de nuestros parques y ciudades (Ramírez et al., 2008). Lo anterior ha derivado en una toma de medidas, por parte de la ciudadanía inconforme (e.g. uso de químicos y redes sin el manejo adecuado) con tal de contrarrestar su presencia en los alrededores de las edificaciones (Giunchi, Soldatini, Vanni, Baldaccini y Albores et al., 2012).

El deterioro en las obras de infraestructura es un aspecto importante a tomar en cuenta; sin embargo, hay pocos estudios que comprueben que las palomas son capaces de transmitir enfermedades a los seres humanos de manera efectiva, algo que ha preocupado al personal universitario que se siente vulnerable a enfermedades por la presencia de las palomas a su alrededor. Hasta el momento, con lo que se cuenta es con investigaciones que evidencian que las palomas contienen aproximadamente unos 60 diferentes patógenos, pero solo siete son 
transmitidos a los seres humanos. Se ha reportado que por lo menos hay cinco patógenos que son virus, nueve tipos de bacterias, 45 tipos de hongos y solo un protozoario. Se encuentra en la lista la Salmonella enterica serotipo Kiambu y uno de los patógenos de transmisión más comunes como lo es Chlamydophila psittaci que produce la clamidia (Haag-Wackernagel et al. 2004). Sin embargo, el hecho de que la paloma sea portadora, no implica que el humano vaya a sufrir un contagio, ya que para una persona saludable el riesgo es muy bajo, inclusive si mantienen un contacto cercano con nidos o pichones. Por el contrario, los pacientes inmuno-deprimidos tienen un mayor riesgo de infección (Haag-Wackernagel et al., 2004).

Una encuesta llevada a cabo en el año 2008 en la provincia de Alajuela, Costa Rica, arrojó que un $60 \%$ de las personas encuestadas piensa que las palomas son transmisoras de enfermedades; sin embargo, un porcentaje igual no conocía a ninguna persona que hubiese sido contagiada por alguna enfermedad asociada directamente a la presencia de las palomas (Ramírez et al., 2008). Esto permite inferir que, el hecho de que las palomas transmitan enfermedades puede que no sea más que una creencia popular o un mito. De ahí la importancia de la elaboración de estudios específicos para determinar científicamente si C. livia es transmisora de agentes potencialmente infecciosos para las personas.

Por todo lo anteriormente expuesto, surgió la motivación de realizar una evaluación del estado de la población en términos de abundancia relativa de la presencia de la paloma de castilla (C. livia). Además, se tomó en cuenta la percepción que posee el personal sobre tal presencia y la problemática de la paloma de Castilla en la Universidad Nacional (UNA), Heredia, Costa Rica.

\section{Materiales y métodos}

\section{Área de Estudio}

Se seleccionaron los edificios del Campus Omar Dengo de la Universidad Nacional, ubicada en la ciudad de Heredia, entre ellos: Física: A (costado oeste), Química: B (costado oeste), Química: C (costado sur), Química: G (costado este), Agrarias: E (costado oeste), Decanato: F (costado oeste) y el edificio de Polímeros: D. El criterio de selección fue: presencia de palomas reportadas por el personal y observación directa del uso de las edificaciones dentro de la Universidad, principalmente en la Facultad de Ciencias Exactas y Naturales.

\section{Métodos}

Durante un periodo de cinco meses se visitaron, tres veces por semana: mañana y tarde (06:00 - 06:30 y 15:00 - 15:30 horas), los edificios de interés. Algunos de los muestreos se realizaron durante las 16:00-16:20 horas con el motivo de conocer los sitios potenciales para el descanso de las aves.

La identificación de las aves fue llevada a cabo en el sitio, mediante observación directa, asimismo se anotó el número de individuos, el uso inmediato de la infraestructura utilizada por las aves (columnas, canoas, tuberías, aire acondicionado, antenas, barandas), comportamiento y, además, si había o no presencia de nido.

Se estimó la abundancia promedio por mes y por edificio de la cantidad de palomas circundantes dentro de los edificios principales donde se ha tenido contacto directo con la presencia de palomas. 
Se aplicó una encuesta semiestructurada de 20 preguntas para obtener resultados que permitieran determinar la situación actual de la convivencia con la paloma de Castilla por parte del personal de la Universidad Nacional. La encuesta se aplicó el viernes 8 de mayo de 2015 a 30 personas en cuatro diferentes escuelas: Química, Matemáticas, Física y Biología, del edificio de las Ciencias Exactas y Naturales.

La aplicación de la encuesta fue realizada por dos personas dentro de las oficinas y laboratorios de dichas escuelas, la dinámica consistió en que el personal fuese entrevistado al mismo tiempo y por separado para minimizar así la influencia que se pudiese desarrollar entre sí. Una vez obtenidos los resultados se procedió a ingresar la información para su posterior análisis, para ello se utilizó el programa estadístico SPSS 15.0.

\section{Resultados}

Se encontró que el costado oeste del edificio de Química (B) presenta el mayor promedio de la abundancia de palomas de Castilla $(4.37 \pm 1.17)$ de manera significativa $(\mathrm{H}=53.15,3,106$; $\mathrm{P}<0.05)$ respecto a los demás edificios (Figura 1 ), mientras que el costado sur de la Escuela de Química (C) obtuvo el menor valor de promedio de la abundancia (1.46 \pm 0.52$)$.

Se comprobó que las palomas de Castilla utilizan el aire acondicionado que se encuentra en costado oeste de Química como un sitio permanente de anidación y se observó que algunos agujeros en el cielorraso de la Escuela de Agrarias son usados por estas como dormitorios.

\section{Percepción social}

Un total de 30 personas fueron encuestadas, se repartió de manera equitativa entre hombres y mujeres. Un 46.7\% se aplicó en la Escuela de Química, 26.7\% en la Escuela de Ciencias Biológicas, y el porcentaje restante entre la Escuela de Matemática y Física, 13.3\% cada una.

\section{Creencias, contacto e información}

Un $66.7 \%$ considera que las palomas producen algún tipo de enfermedad a los humanos; el porcentaje restante que no. Un $63.3 \%$ no tiene contacto directo con ellas y un $23.3 \%$ considera que sí tiene contacto de manera indirecta; solo un $13.3 \%$ tiene contacto directo. Un $86.7 \%$ nunca las ha visto en sus oficinas y un $13.7 \%$ si las ha encontrado en alguna ocasión dentro. Con respecto a recibir información por parte de salud laboral, un $63.3 \%$ sí ha recibido y un $36.7 \%$ no.

\section{Erradicación}

De las 30 personas encuestadas un total de 53,3\% están en desacuerdo con la erradicación de la paloma de Castilla en la Universidad Nacional, sin embargo, un total de 46,7\% está de acuerdo. El 60\% de las mujeres encuestadas apoyaría ante un eventual proceso de erradicación de la paloma de Castilla mientras que solo un 33.3\% de los hombres encuestados estaría a favor.

\section{Erradicación por escuelas}

La Escuela de Física, a pesar de no presentar problemas de presencia de palomas, un 100\% estaría de acuerdo $(n=8)$ en un eventual proceso de erradicación, no es así para la Escuela de Química, que es la que presenta el mayor promedio de la abundancia por la presencia de estos individuos (un 57.1\% estaría de acuerdo, un 42.9\% no estaría de acuerdo). 
ISSN Electrónico: 2215-3470

DOI: http://dx.doi.org/10.15359/ru.31-1.4
UNICIENCIA Vol. 31, No. 1, pp. 29-38. Enero-junio, 2017.

URL: www.revistas.una.ac.cr/uniciencia Email: revistauniciencia@una.cr

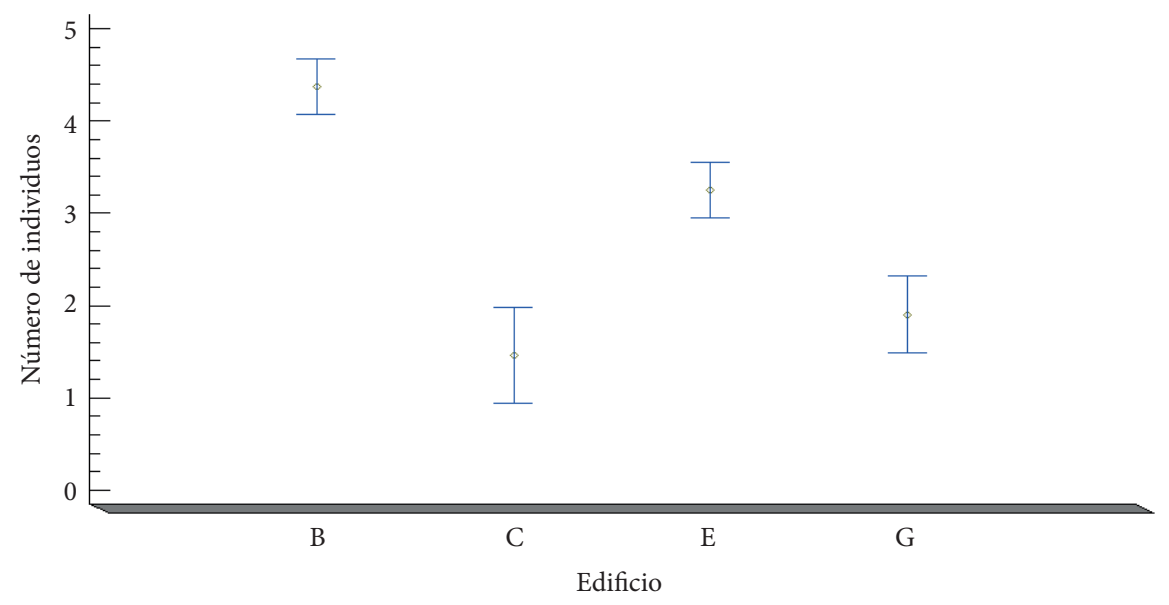

Figura 1. Estimación de las medias de la abundancia de Columba livia por edificios dentro de la Universidad Nacional, Heredia, Costa Rica. 2015. Elaboración propia del estudio.

Respecto a los promedios por mes, junio presenta la menor cantidad de individuos (2.33 \pm 0.58 ), y durante los meses de febrero, marzo, abril y mayo la cantidad de individuos en promedio se mantuvo similar $(\mathrm{H}=1.17 ; 4,105 ; \mathrm{P}>0.05)$ (Figura 2).

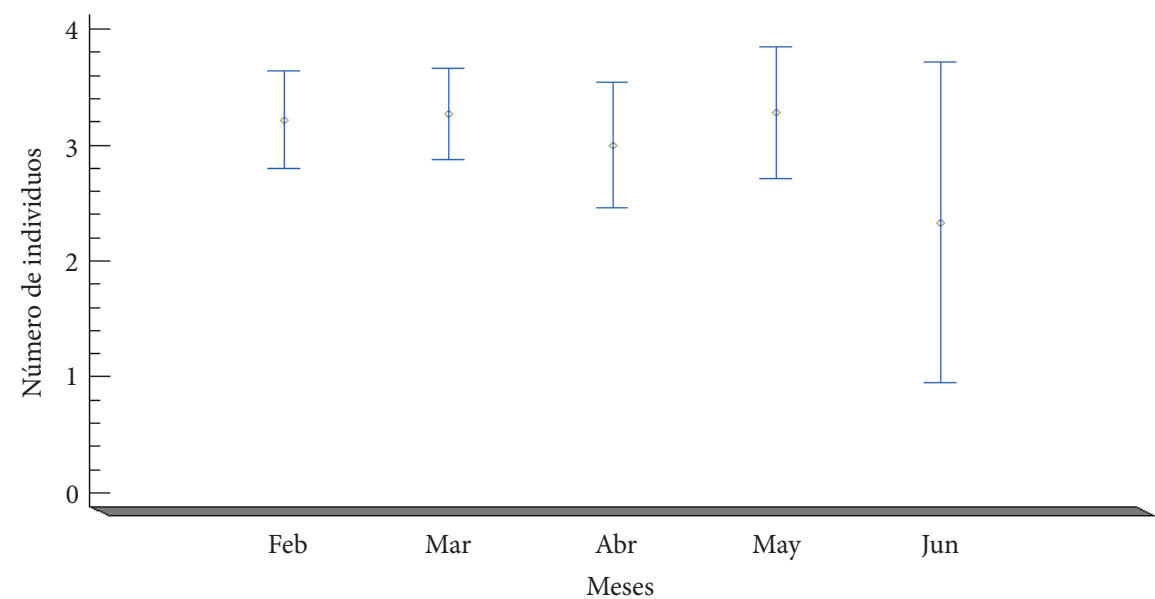

Figura 2. Promedios mensuales observados de Columba livia en los edificios dentro de la UNA. 2015. Elaboración propia del estudio.

De las personas encuestadas, un $63.3 \%$ se siente satisfecha con la limpieza de las oficinas en contraste con el $36.7 \%$ restante. Cuando se les consultó si han presentado alergias asociadas con la condición laboral un $36.7 \%$ menciona que sí; mientras que el $63.3 \%$ restante menciona que no, porcentajes compartidos con el grado de satisfacción de la limpieza de oficinas. Las alergias respiratorias las han presentado funcionarios de la Escuela de Química y Física. 
UNICIENCIA Vol. 31, No. 1, pp. 29-38. Enero-junio, 2017.

ISSN Electrónico: 2215-3470

URL: www.revistas.una.ac.cr/uniciencia

DOI: http://dx.doi.org/10.15359/ru.31-1.4

\section{Discusión}

\section{Problemática}

La presencia de palomas de Castilla en el hábitat urbano y su grado de interacción con la vida y las actividades humanas se puede percibir en muchas maneras que van, desde las aves inofensivas y dóciles hasta plagas dañinas, dependiendo del bagaje cultural de cada persona (Jerolmack, 2008, Ramírez et al., 2008).

Johnston y Janiga (1985) afirman que las palomas domésticas tienen una formidablecapacidad para convertirse en plaga. Algunos de los factores que se han identificado como importantes para convertirse en una plaga incluyen las principales características de las palomas, como ser granívoro, tener un cultivo alimentario de almacenamiento, alta tasa reproductiva, hábitos coloniales y forrajeo en grupo. En la Universidad Nacional esos reservorios fueron identificados principalmente en las zonas de las Escuelas de Química y Agrarias, donde la infraestructura como canoas, techos, barandas y aire acondicionado son utilizados por las palomas como sitios de descanso, forrajeo y anidación.

Por otra parte, Haag-Wackernagel (1995) y Sacchi, Gentilli, Razzetti y Barbieri (2002) enuncian que la abundancia de infraestructura y los recursos alimentarios son factores ecológicos claves para atraer palomas en la mayoría de ciudades y pueblos de todo el mundo. La universidad, por su cercanía con parques como el central de Heredia, donde hay presencia de dichas palomas, no está exenta de siempre tener cierta cantidad de individuos en sus instalaciones. Mientras no se haga manejo adecuado de algunas infraestructuras, seguirán siendo sitios potenciales para albergar a estos individuos.

\section{Daños a la infraestructura}

Tal como lo afirman Pimentel, Lach, Zuniga y Morrison (2000), un factor que contribuye con la relación negativa entre las palomas y los seres humanos es el problema de los daños que sufren los edificios de las zonas urbanas, ya que, por medio de las acumulaciones de excretas, causan daños estructurales y estéticos en los edificios, acelerando su deterioro y el aumento en costes de mantenimiento. En los edificios de las Escuelas de Química y de Agrarias se observó presencia de heces, producto de la aglomeración de algunos individuos, donde utilizan estructuras como barandas, tuberías y columnas para descansar y acicalarse (Figura 3); otras estructuras que son muy utilizadas por las palomas son las canoas, techos y aires acondicionados de las Escuelas de Química y Agrarias, las cuales son usadas como lugares para cópula, descanso y anidación (Figura 4).

Se ha demostrado que los edificios constituyen un sitio ideal para la anidación y el descanso de las palomas de Castilla, lo que contribuye directamente en el crecimiento de sus poblaciones (Ballarini, Baldaccini, y Pezza, 1989; Sacchi et al., 2002). Asimismo, de acuerdo con Lancaster y Rees (1979), los edificios que más utilizan las palomas son aquellos que poseen agujeros, cavidades y salientes donde pueden desarrollar algunas de sus funciones vitales. Algunos edificios donde se identificó este problema fueron los de la Escuela de Química, específicamente los costados este, oeste y sur, además del costado oeste de la Escuela de Agrarias (Figura 5) que, debido a los agujeros presentes en el cielorraso son un lugar idóneo para el descanso y anidación de palomas. 
Por lo anterior, es recomendable tomar algunas acciones de manejo a corto y largo plazo. Entre ellas se sugiere: informar a la ciudadanía acerca de métodos para mejorar la eliminación de los residuos reduciéndose de esta manera el suministro de alimentos; reducir los sitios potenciales de anidación, perchado y descanso mediante la modificación del hábitat (Olalla, Ruiz, Ruvalcaba y Mendoza, 2009), esta última estrategia consiste en no permitirles el acceso a la estructura por medio de la protección de los edificios: usos de vallas, redes, entre otros.

En caso de darse un aumento significativo en los edificios, se recomienda eliminar todos los nidos de manera periódica; esta acción deberá estar contemplada demostrando con datos (estudios de seguimiento) el incremento. Lo anterior disminuiría considerablemente la población a largo plazo, donde acciones de control no han tenido efecto (Giunchi et al., 2012). Otro aspecto importante de mencionar es que no hubo un incremento del número de palomas a través de todo el estudio, por lo que es posible que las mismas especies depredadoras que existen dentro y en los alrededores de la Universidad estén fungiendo como controladores naturales. En dos ocasiones se observó al Buteo plagiatus (gavilán gris), depredando palomas de Castilla dentro de la Universidad, por lo que la permanencia de estas especies resulta de importancia para mantener en regulación de algunos grupos de vertebrados.

Es importante entender que estas poblaciones de palomas son muy dinámicas, por lo que es de esperar que algunos individuos se puedan trasladar a otros edificios en donde encuentren las condiciones idóneas para establecerse (sitios de percha, establecimientos planos con sombra para colocar los nidos, huecos en los tejados entre otras. Asimismo, Barlow, Kean y Briggs, (1997) menciona que el sacrificio en especies monógamas con una alta tasa de mortalidad y productividad, como lo son las palomas de Castilla, no es un método efectivo para el control de las poblaciones, además, ese tipo de métodos no es bien aceptado por la ciudadanía, lo que podría provocar un aumento en los problemas de carácter ético.
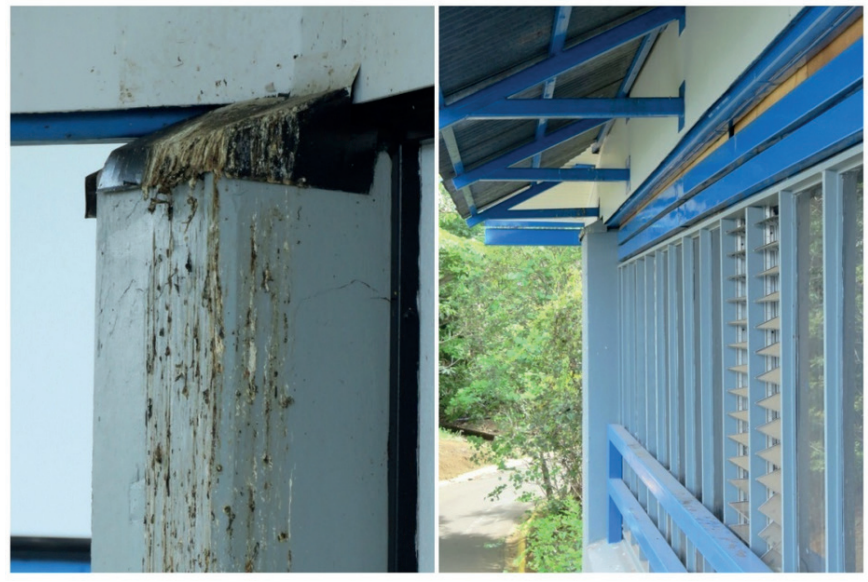

Figura 3. Acumulación de excretas de la Paloma de Castilla en la Universidad Nacional, Heredia, Costa Rica, 2015. 
UNICIENCIA Vol. 31, No. 1, pp. 29-38. Enero-junio, 2017.

URL: www.revistas.una.ac.cr/uniciencia

Email: revistauniciencia@una.cr

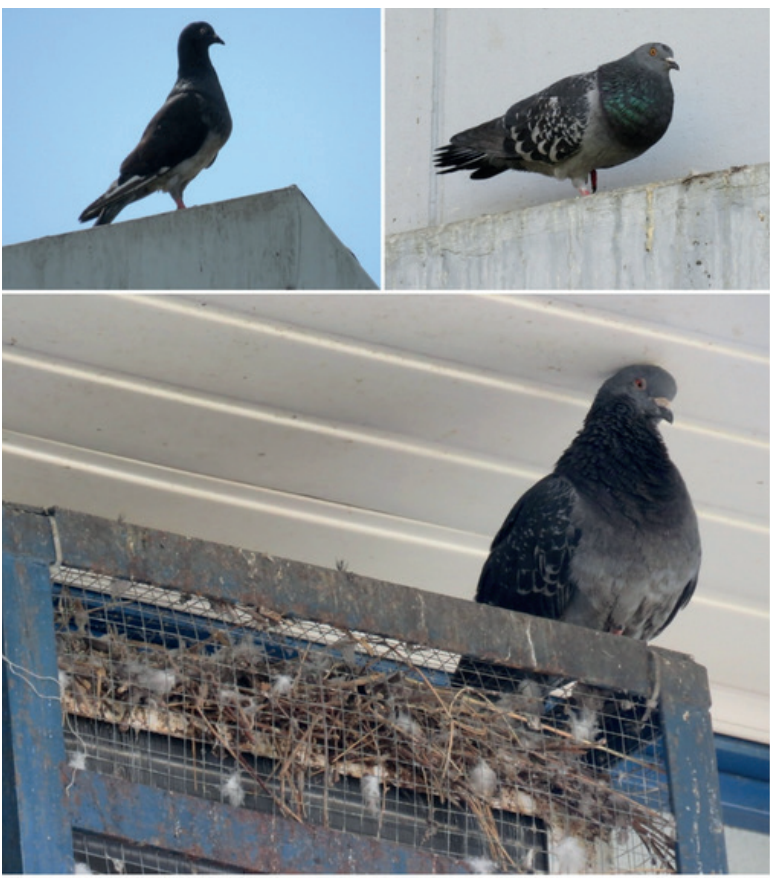

Figura 4. Sitios potenciales para percha y anidación de la paloma de Castilla en la Universidad Nacional, Heredia, Costa Rica. 2015.
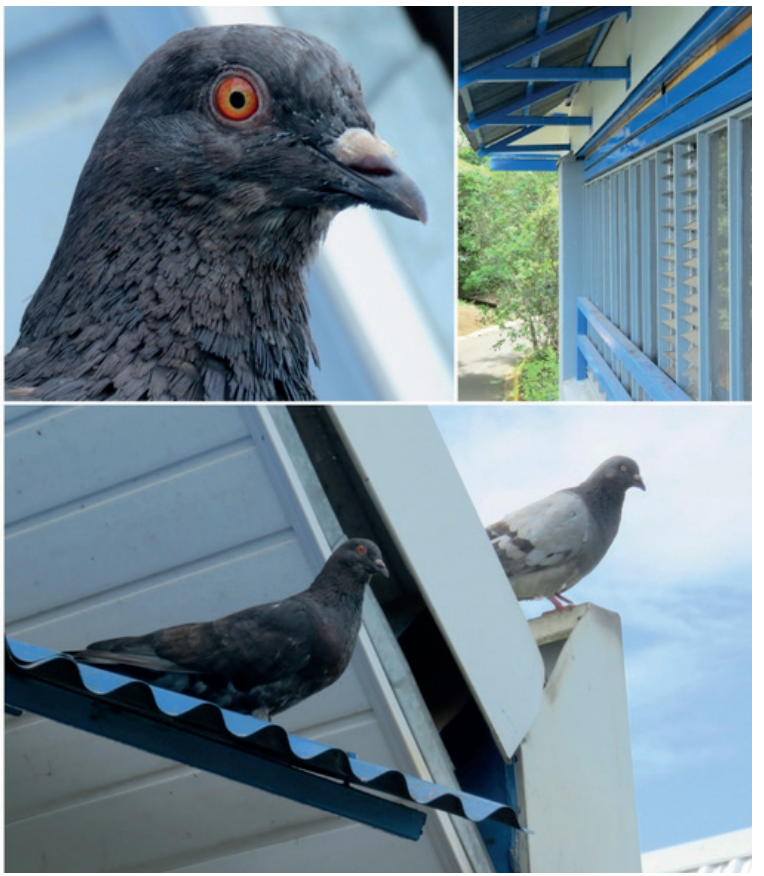

Figura 5. Infraestructura y cavidades de uso potencial para las palomas de Castilla en la Universidad Nacional, Heredia, Costa Rica. 2015.

Es importante llamar la atención sobre la necesidad de que exista una coordinación con mantenimiento de la Universidad, para que dé soporte inmediato para reparar huecos en los tejados o remover los sitios potenciales, donde se observe la presencia de las palomas de Castilla, y no confundir otras poblaciones existentes en los edificios que no causan molestia (búhos, y otras especies de aves).

\section{Recomendaciones finales}

\section{Acciones a corto plazo}

1. Reducir sitios artificiales de percha para la paloma de Castilla mediante el uso de redes especiales.

2. Mantener sin agujeros o cavidades los cielorrasos y techos de la infraestructura universitaria.

3. Eliminar los nidos periódicamente (cada seis meses) mediante la extracción manual de estos mismos.

4. Eliminar la acumulación de excretas al menos cada seis meses por medio del lavado a presión. 
ISSN Electrónico: 2215-3470

DOI: http://dx.doi.org/10.15359/ru.31-1.4
UNICIENCIA Vol. 31, No. 1, pp. 29-38. Enero-junio, 2017.

URL: www.revistas.una.ac.cr/uniciencia Email: revistauniciencia@una.cr

\section{Acciones a largo plazo}

1. Educar a la población acerca de los problemas que causa la mala gestión de los residuos y del suministro de recursos alimentarios.

2. Llevar a cabo estudios sobre ecología y dinámica de las poblaciones para entender cómo las palomas usan el hábitat urbano.

3. Desarrollar investigación microbiológica para saber cuáles enfermedades pueden estar presentes en las heces que se encuentran sobre la infraestructura

\section{Agradecimientos}

Al Decanato de la Facultad de Ciencias Exactas y Naturales, Miembros Consejo Académico de la Facultad de Ciencias Exactas y Naturales y en especial a Señor Felipe Reyes Solares, por el apoyo en las gestiones de este proyecto.

\section{Referencias}

Ballarini, G., Baldaccini, N. E., y Pezza, F. (1989). Colombi in città: aspetti biologici, sanitari e giuridici: metodologie di controllo. Istituto nazionale di biologia della selvaggina: Alessandro Ghigi.

Barlow, N. D., Kean, J. M., \& Briggs, C. J. (1997). Modelling the relative efficacy of culling and sterilisation for controlling populations. Wildlife Research, 24(2), 129-141. http://dx.doi.org/10.1071/WR95027

Giunchi, D., Soldatini, C., Vanni, L., Baldaccini, N. E., y Albores-Barajas, Y. V. (2012). Feral pigeons: problems, dynamics and control methods. INTECH Open Access Publisher. http://dx.doi. org/10.5772/31536

Haag-Wackernagel, D. (1995). Regulation of the street pigeon in Basel. Wildlife Society Bulletin, 23 (2), 256-260.

Haag-Wackernagel, D. y Moch, H. (2004). Health hazards posed by feral pigeons. Journal of Infection, 48(4), 307-313. http://dx.doi.org/10.1016/j.jinf.2003.11.001

Jerolmack, C. (2008). How pigeons became rats: The cultural-spatial logic of problem animals. Social Problems, 55(2), 72-94. http://dx.doi.org/10.1525/sp.2008.55.1.72

Johnston, R.F., y Janiga, M. (1985). Feral pigeons, Oxford University Press, New York.

Jokimaki, J., y Suhonen, J. (1998). Distribution and habitat selection of wintering birds in urban environments. Landsc. Urban Plann, 34, 253-263. http://dx.doi.org/10.1016/S0169-2046(97)00089-3

Lancaster, R. K., y Rees, W. E. (1979). Bird communities and the structure of urban habitats. Canadian Journal of Zoology, 57(12), 2358-2368. http://dx.doi.org/10.1139/z79-307

Mendez-Tovar, L. J.; Mainou, L. M.; Pizarro, S. A.; Fortoul-Vandergoes, T. y Lopez- Martinez, R. (1995). Fungal biodeterioration of colonial facades in Mexico City. Revista Mexicana de Micologia, 11, 133-144.

Olalla, A., Ruiz, G., Ruvalcaba, I., y Mendoza, R. (2009). Palomas, especies invasoras. Biodiversitas, 82, 7-10.

Pimentel, D., Lach, L., Zuniga, R., y Morrison, D. (2000). Environmental and economic costs of nonindigenous species in the United States. BioScience, 50(1), 53-65. http://dx.doi. org/10.1641/0006-3568(2000)050[0053:EAECON]2.3.CO;2 
UNICIENCIA Vol. 31, No. 1, pp. 29-38. Enero-junio, 2017.

ISSN Electrónico: 2215-3470

URL: www.revistas.una.ac.cr/uniciencia

DOI: $\underline{\text { http://dx.doi.org/10.15359/ru.31-1.4 }}$

Email: revistauniciencia@una.cr

Ramírez, O., Amador, M., Camacho, L., Carranza, I. J., Chaves, E., Moya, A., y Quiros, W. (2008). Conocimiento popular de la paloma de Castilla (Columba livia) en el Parque Central de Alajuela. Zeledonia, 12(1), 14-19.

Sacchi, R., Gentilli, A., Razzetti, E., y Barbieri, F. (2002). Effects of building features on density and flock distribution of feral pigeons Columba livia var. domestica in an urban environment. Canadian Journal of Zoology, 80(1), 48-54. http://dx.doi.org/10.1139/z01-202

\section{(C) $\oplus \Theta \Theta$}

Evaluación y percepción de la presencia de palomas Columba livia (Columbiformes:Columbidae) en la Facultad de Ciencias Exactas y Naturales de la Universidad Nacional de Costa Rica durante el primer semestre de 2015 (Oscar Ramírez-Alán y otros) por Revista Uniciencia se encuentra bajo una Licencia CreativeCommons Atribución-NoComercial-SinDerivadas 3.0 Unported. 